\title{
Reading and Listening Comprehension Subskills: The Match between Theory, Coursebooks, and Language Proficiency Tests
}

\author{
Aynur Ismayilli Karakoc* \\ School of Linguistics and Applied Language Studies, Victoria University of Wellington, New Zealand
}

Corresponding Author: Aynur Ismayilli Karakoc, E-mail: phdvuw@gmail.com

\section{ARTICLE INFO}

Article history

Received: March 10, 2019

Accepted: June 24, 2019

Published: August 31, 2019

Volume: 10 Issue: 4

Advance access: July 2019

Conflicts of interest: None

Funding: None

\section{Key words:}

Reading Subskills,

Listening Subskills,

Skills in Coursebooks,

Skills in Tests

\begin{abstract}
Different theoretical and empirical taxonomies of reading and listening comprehension (RC, LC) are available in the literature. Most of language tests and tasks in English as foreign or second language (EFL/ESL) coursebooks are based on the classifications of reading and listening subskills (micro-skills) offered in theory. However, these taxonomies have not cross-checked whether the theoretical subskills are practiced in ESL/EFL coursebooks and assessed in proficiency tests. Nor have they listed the shared and exclusive RC and LC subskills in a unified fashion. For this purpose, theoretical subskills offered in Applied Linguistics literature, nine internationally popular EFL/ESL proficiency tests and 25 widely used coursebook tasks were collected, cross-compared, repetitions were eliminated, and a final inclusive list of common and exclusive subskills was prepared. The findings suggested ten common reading and listening subskills. The subskills exclusive to reading were seven, and exclusive to listening were four. This list is hoped to be helpful for teachers in developing their own tests, and the coursebook developers in preparing the content materials.
\end{abstract}

\section{INTRODUCTION}

Language ability is manifested through reading and listening skills. These are also called receptive skills comprising different and similar processes. There are modality unspecific and modality-specific perspectives explaining the comprehension ability (Schroeders, Wilhelm \& Bucholtz, 2010). According to a modality unspecific or single skill view, a unique single factor can explain the comprehension, implying that reading comprehension (RC) and listening comprehension (LC) may comprise the same cognitive processes. On the other hand, a modality-specific or multiple skill view posits that various factors can explain RC and LC with some differences and some similarities. This dichotomy also corresponds with the unitary and dual comprehension models (Lund, 1991). The unitary model posits that a single comprehension process functions for reading and listening, while the dual model holds that there are both differences and similarities between modalities.

In terms of differences, it is believed that listening is more cognitively demanding than reading (Buck, 2001) because:

(a) it requires more attention to sounds and prosodic features,

(b) it occurs in real time, and listeners have less control on the input, and do not have the opportunity to check,

(c) speech is unplanned having pauses, false starts, hesittions and so forth (Vandergrift, 2006; Wagner, 2013), (d) it has different speeds of input, use of cognates, reductions, blending of sounds and back-channel cues,

(e) speech is shorter than written units with vaguer and more colloquial language (i.e., there are more pronouns, redundancies, fillers, self-corrections, less standard grammar in speech, and conjunctions are used instead of subordination), and

(f) meaning is conveyed by gestures and body language in speech (Brown, 2011).

Regarding the similarities, both receptive skills involve decoding and comprehension using language and background knowledge. As Alderson (2000) suggested, reading is a cognitive problem-solving activity whereby it is also applicable to listening. Both LC and RC utilize similar cognitive processes (bottom-up, top-down, and integrative) (Hirai, 1999; Powers, 2013), and abilities (Spies, 2011). Also, it is argued that receptive skills may share common elements such as vocabulary, sentence patterns, idea organization, adjustment to the language function (Hollingsworth, 1968), or similar features utilizing the skills such as understanding, comprehending, analyzing, synthesizing, interpreting, and evaluating the input (Emiroğlu \& Pınar, 2013). In other words, both RC and LC can have similarities or shared traits, as both are receptive skills. Nevertheless, to some extent, they are also different in that they have different cognitive loads and linguistics features. 
The picture becomes more complex because there are various lists of subskills, some of which could be called 'armchair' perspectives (Alderson, 2000). Different language proficiency tests and language coursebooks are built on language skills. Therefore, it is important to investigate what kind of reading and listening subskills are included in coursebooks, and what subskills are tested in language proficiency tests. It is hoped that examining the subskills in these sources would yield a more inclusive result. Moreover, it would elicit preliminary ideas on whether those subskills are shared or unique. It is hoped that the collective list would help teachers in developing their own tests, preparing lesson materials, and the coursebook designers working on the content of the materials. Motivated by this perspective, the aim of this study is to investigate reading and listening subskills across theories, language proficiency tests, and language coursebooks.

\section{LITERATURE REVIEW}

The reading literature has suggested different reading models including the componential and process models (Urquhart \& Weir, 1998). These models also correspond with the 'reading processes and 'reading product' identified by Alderson (2000). The componential model describes reading in terms of certain factors, while the process model may explain how these factors operate. The process model deals with the reader-text interaction involving top-down, bottom-up and interactional models, while the componential model focuses on the reading product and skills or knowledge involved in that product.

The literature predominantly suggests that reading is at least two-dimensional (Urquhart \& Weir, 1998) although, in the current era, this premise is superseded by multidimensional models. The review of the literature showed that applied linguists explained reading in terms of its (a) components, (b) types, and (c) subskills. As summarized in Koda (2005), different componential models have been put forth, if not all empirically validated, including the two-component model (i.e., lower level decoding and higher-level linguistic comprehension); the three-component model (i.e., conceptual abilities, process strategies and background knowledge), and another three-componential model (i.e., language, literacy, and background knowledge). To explain how learners understand large amounts of texts, different reading types, including careful and expeditious reading at global and local levels have been suggested (Unaldi, 2004; Urquhart \& Weir, 1998; Weir, 2005). Finally, there is a growing number of studies explaining reading in terms of its underlying subskills. In this research, reading was explained by splitting this process into component skills (Grabe, 1991), or interrelated and interdependent subskills, which can also be applied to listening, speaking and writing (Cummins, 2014).

The listening literature shows that LC has been distinguished under the 'general' and 'comprehension' models by Aryadoust (2013). The general model is mainly based on L1 cognitive psychology studies representing listening processes, including bottom-up, top-down, and interactive processes. Like RC, the role of both approaches in listening input processing is emphasized since LC is an outcome of communication between linguistic and general knowledge (Buck, 2011). Moreover, a combination of these approaches is important to validate listening ability tests (Rost, 2011). This is because it fits individual learning styles (Flowerdew \& Miller, 2005), and is necessary for listening skill curriculum for effective teaching (Hinkel, 2006). Similar to RC, LC is also accepted as a multidimensional trait containing multiple divisible constituents (Aryadoust, 2013). Consequently, multiple dimensions lead to a varying number of subskills reflected in the aforementioned speculated and research-based taxonomies.

Although there is no consensus on the number of subskills, the multi-divisibility of reading and listening has most intrigued the researchers leading to a varying number of subskills. Subskills have been proposed either as a result of qualitative and quantitative empirical investigations (e.g., Buck, Tatsuoka \& Kostin, 1997; Eom, 2006; 2008; Goh \& Aryadoust, 2015; Jang, 2005; Kim, 2011; Powers, 1985; Song, 2008), or theories (Brown, 2004; Hughes, 2003; Richards, 1983; Weir, 1993). Literature shows that Northern American researchers (Eom, 2006; 2008; Jang, 2005; Kim, 2011; Song, 2008; and others) explained the multidimensionality in terms of particular subskills or language attributes, while British researchers and followers (Khalifa \& Weir, 2009; Shiotsu, 2010; Weir, Huizhong \& Yang, 2000) have mostly premised the subskills on classification of reading types classified by Urquhart and Weir (1998). Regardless of the definitions and techniques, it can be concluded that the types, abilities, skills, micro-skills explain the construct of reading (Weir, Hawkey, Green, Unaldi \& Devi, 2009) and listening. Therefore, it is important to arrive at a complex picture of the subskills from pedagogic and theoretical perspectives. Considering that the majority of language tests and tasks in language coursebooks are based on the classification of language skills offered in applied linguistics, it is necessary to arrive at a unifying picture by identifying the match between these skills mentioned in different sources. The study aims to answer:

What are the subskills shared between and exclusive to reading and listening comprehension mentioned in applied linguistics theory and applied to language tests and coursebooks?

\section{METHOD}

Language pedagogy, tests, and theories are interrelated; therefore, it is necessary to identify the subskills included in these sources to understand the complexity of them. First, the subskills mentioned in the literature of applied linguistics were checked. These skills were mentioned in theoretical and empirical papers (for details, see Richards, 1983; Buck \& Tatsuoka, 1998; Hughes, 2003; Brown, 2004; Eom, 2006; 2008; Kim, 2011).

Second, the manuals of nine EFL/ESL tests (Test of English as a Foreign Language - Internet-Based Test (TOEFL IBT), International English Language Testing System (IELTS), First Certificate in English (FCE), Cambridge English: Advanced (CAE), Cambridge English Proficiency (CPE), Pearson Test of English (PTE) Academic, Canadian 
Table 1. The list of subskills shared between and exclusive to RC and LC

\begin{tabular}{ll}
\hline Common & Understanding a main idea and general information \\
Subskills Shared & Understanding facts, details and specific information \\
between & Understanding a writer's or speaker's attitude and purpose \\
RC and LC & Inferring a meaning of an unknown word from the context \\
& Inferring indirect information from the context \\
& Summarizing a message or information \\
& Recognizing cause-effect or comparison relations \\
& Paraphrasing information \\
& Understanding the function of words or phrases in the context \\
Transferring information to pictures, maps, tables, or diagrams
\end{tabular}

Subskills Exclusive

to $\mathrm{RC}$

Subskills Exclusive

to LC
Identifying a referent word in a text

Completing a sentence or paragraph with missing words or phrases

Matching headings to a paragraph

Choosing an appropriate title for a text

Inserting a sentence into a gap in a text

Translating a sentence into a native language

Recognizing or using grammar or grammar points in a context

Identifying an error in a transcription

Predicting the end of the continuation of a message or history

Perceiving individual sounds

Listening to and ordering statements according to the message
Academic English Language Assessment (CAEL), MELAB (Michigan English Language Assessment Battery) and Examination for the Certificate of Competency in English (ECCE)) were analyzed, and subskills attempted in these tests were listed (Appendix 1). These tests were consulted because they are popular international tests.

Third, the tasks in five mostly used EFL/ESL coursebooks published by the UK and US publishers, including "New English File”, "Face2Face, "New Inside Out", "Outcomes", "Language Leader" with all available levels were listed (Appendix $2 \& 3$ ). These books were chosen considering that they are the most widely used series at universities to train the students in English. Only the tasks (exercises) under reading and listening sections were checked, and the ones under different sections, such as pronunciation or grammar were not included in the list.

Fourth, the most frequent RC and LC subskills documented in applied linguistics literature, EFL/ESL proficiency tests, and coursebook tasks were also tallied. Finally, the items in these taxonomies were cross-checked, repetitions were eliminated, and a list of subskills shared between RC and LC and those exclusive to RC and LC were prepared (Table 1). These lists were also cross-checked and agreed by other 2 experienced EFL teachers with a Master's degree in ELT.

\section{RESULTS AND DISCUSSION}

This study attempted to prepare a taxonomy of the subskills shared between and exclusive to RC and LC. For this purpose, the subskills documented in Applied Linguistics theories, applied to different tests, and practiced in coursebooks were listed, and a final conclusive taxonomy was prepared. As shown in Table 1, the agreed number of common subskills was ten, while subskills unique to reading were seven. The subskills unique to listening were four. This study is in line with previous empirical findings in that RC and LC are divisible containing several subskills or micro-skills (Eom, 2006; 2008; Goh \& Aryadoust, 2015; Kim, 2011 and others). Yet, this study can be considered unique as it attempted to offer common and exclusive RC and LC subskills.

Research shows that EFL teachers, test developers, syllabus designers have benefited from multidimensional employment of reading ability to design exercises and test tasks (Grabe, 1991; Jordan, 1997; Urquhart \& Weir, 1998; Weir, Huizhong, \& Yan, 2000; Khalifa \& Weir, 2009). Therefore, this taxonomy could be helpful for teachers and material developers because this inclusive list offers operationalized subskills. Moreover, because subskills are helpful for diagnostic purposes (Alderson, 2005; Field, 1998; Goh \& Aryadoust, 2015), this taxonomy may help teachers or teacher-researchers to diagnose the learners' strengths and weaknesses on subskills. To add further, since students themselves view their academic reading as multidimensional (Weir et al., 2009), this taxonomy may help researchers or teachers to design self-assessment tests or tools based on the subskills provided here.

To follow up this study, it is necessary to design $\mathrm{RC}$ and LC tests with the offered subskills. It is also necessary to observe the students while answering test questions on the measured subskills. The test scores, students' think-aloud protocols and the interviews could further explain the dimensionality of the subskills. This would also explain learners' performance in subskills common to both RC and LC.

\section{LIMITATIONS}

Nevertheless, this study has some limitations. Subskills in diagnostic tests could have been investigated as well. 


\section{CONCLUSION}

The study's purpose was to investigate the subskills in reading and listening comprehension. For this purpose, subskills in theory, language proficiency tests and coursebooks were listed. After rigorous analyses of the data, a list of conclusive subskills was prepared. In the next stage, the taxonomy was refined further to investigate the common and exclusive subskills. The results showed that $\mathrm{RC}$ and $\mathrm{LC}$ are not unitary and both consist of similar and different subskills. This taxonomy offers a list of operational subskills; therefore, it may be helpful for teachers and content developers to design tests and prepare course materials.

\section{ACKNOWLEDGEMENT}

This report is part of my MA thesis study. I thank Dr. Hossein Farhady for his guidance during the study. I thank the editors for their feedback.

\section{REFERENCES}

Alderson, J. C. (2000). Assessing reading. Cambridge: Cambridge University Press.

Alderson, J. C. (2005). Diagnosing foreign language proficiency: The interface between learning and assessment. London, UK: Continuum.

Aryadoust, V. (2013). Building a validity argument for a listening test of academic proficiency. UK: Cambridge Scholars Publishing e-book.

Brown, H. D. (2004). Language assessment: Principles and classroom practices. NY: Pearson Education.

Brown, J. D. (2008). Principal components analysis and exploratory factor analysis: Definitions, differences, and choices. JALT Testing \& Evaluation SIG Newsletter, 13 (1), 26-30.

Brown, S. (2011). Listening myths: Applying second language research to classroom teaching. US: University of Michigan Press.

Buck. G., Tatsuoka, K., \& Kostin, I. (1997). The subskills of reading: Rule-space analysis of a multiple-choice test of second language reading comprehension. Language Learning, 47 (3), 423-466.

Buck. G. (2001). Assessing listening. Cambridge: Cambridge University Press.

CAEL Test. Retrieved from: www.cael.ca/edu/format.shtml

Cambridge CAE Test. Retrieved from: www.cambridgeenglish.org/exams/advanced/whats- in-the-exam

Cambridge CPE Test. Retrieved from: www.cambridgeenglish.org/exams/proficiency/whats- in-the-exam

Cambridge FCE Test. Retrieved from: www.cambridgeenglish.org/exams/first/whats-in-the-exam

Cotton, D., Falvey, D., \& Kent, S. (2008). Language leader coursebook: Upper-intermediate. England: Pearson Education.

Cotton, D., Falvey, D., \& Kent, S. (2008). Language leader coursebook: Intermediate. England: Pearson Education.

Cotton, D., Falvey, D., Kent, S., Lebeau, I., \& Rees, G. (2010). Language leader coursebook: Advanced. England: Pearson Education.
Cunningham, G., Bell, J., \& Redston, C. (2009). Face2face: Advanced student"s book. Cambridge: Cambridge University Press.

Cummins, J.(2000). Language, power and pedagogy: Bilingual children in the crossfire. Clevedeon, UK: Multilingual Matters.

Dellar, H., \& Walkley, A. (2010). Outcomes: Upper-intermediate student"s book. UK: Heinle, Cengage Learning.

Dellar, H., \& Walkley, A. (2010). Outcomes: Intermediate student"s book. UK: Heinle, Cengage Learning.

Dellar, H., \& Walkley, A. (2010). Outcomes: Pre-intermediate student"s book. UK: Heinle, Cengage Learning.

Dellar, H., \& Walkley, A. (2011). Outcomes: Elementary student"s book. UK: Heinle, Cengage Learning.

Dellar, H., \& Walkley, A. (2012). Outcomes: Advanced student"s book. UK: Heinle, Cengage Learning.

ECCE Information Pack. Retrieved from www.cambridgemichigan.org/ecce

Emiroğlu, S. \& Pınar, F. N. (2013). Dinleme becerisinin diğer beceri alanlari ile ilişkisi. Turkish Studies - International Periodical for The Languages, Literature and History of Turkish or Turkic 8 (4), 769-782.

Eom, M. (2006). An investigation of operationalized constructs of second language listening tests (Doctoral dissertation). The University of Iowa, USA. Retrieved from ProQuest (UMI Number: 3229656).

Eom, M. (2008). Underlying factors of MELAB listening constructs. Spaan Fellow Working Papers in Second or Foreign Language Assessment, 6, 77-94. Ann Arbor, MI: University of Michigan English Language Institute.

Field, J. (1998). Skills and strategies: Towards a new methodology for listening. ELT Journal, 52 (2), 110-118, Oxford University Press.

Flowerdew, J., \& Miller, L. (2005). Second language listening: Theory and practice. New York: Cambridge University Press.

Goh, C. C., \& Aryadoust, V. (2014). Examining the Notion of Listening Subskill Divisibility and its Implications for Second Language Listening. International Journal of Listening.

Grabe, W. (1991). Current developments in second language reading research. TESOL Quarterly, 25 (3), 375-406.

Hinkel, E. (2006). Current perspectives on teaching the four skills. TESOL Quarterly, 40 (1), 109-131.

Hirai, A. (1999). The relationship between listening and reading rates of Japanese EFL learners. The modern language journal, 83 (3), 367-384.

Hollingsworth, P. M. (1968). Interrelating listening and reading. Paper presented at International Reading Association Conference: Boston.

Hughes, A. (2003). Testing for language teachers. Cambridge: Cambridge University Press.

IELTS. Retrieved from www.ielts.org/test_takers_information/question_types.aspx

Jang, E. E. (2005). A validity narrative: Effects of reading skills diagnosis on teaching and learning in the context of NG TOEFL. (Doctoral dissertation). University of Illinois at Urbana-Champaign, USA. Retrieved from ProQuest (UMI Number: 3182288). 
Jennings, M., Fox, J., Graves, B., \& Shohamy, E. (1999). The test-takers' choice: an investigation of the effect of topic on language-test performance. Language Testing, 16 (4), 426-456.

Jordan, R. R. (1997). English for Academic Purposes: $A$ Guide and Resource Book for Teachers. Cambridge: Cambridge University Press.

Kay, S., \& Jones, V. (2008). New inside out: Pre-intermediate student"s book. Oxford: MacMillan Education.

Kay, S., \& Jones, V.(2009). New inside out: Intermediate student"s book. Oxford: Macmillan Education.

Kay, S., \& Jones, V. (2009). New inside out: Upper-intermediate student"s book. Oxford: MacMillan Education.

Kay, S., Jones, V., Gomm, H., Seymour, D., Brown, C., \& Dawson, C. (2010). New inside out: beginner teacher"s book. Oxford: Macmillan Education.

Kay, S., Jones, V., Gomm, H., Maggs, P., \& Dawson, C. (2010). New inside out: Advanced teacher"s book. Oxford: Macmillan Education.

Khalifa, H., \& Weir, C. J. (2009). Studies in language testing 29: Examining reading: research and practice in assessing second language reading. Cambridge: Cambridge University Press.

Kim, A. Y. (2011). Examining second language reading components in relation to reading test performance for diagnostic purposes: A fusion model approach. (Doctoral dissertation). Teachers College, Columbia University, USA. Retrieved from ProQuest (UMI Number: 3484246).

Kimzin, G., \& Proctor, S. (1986). An ELI academic listening comprehension needs assessment: Establishing goals, objectives, and microskills. Unpublished manuscript. HI: University of Hawaii at Manoa.

Koda, K. (2005). Insights into second language reading. A cross-linguistic approach. New York, NY: Cambridge University Press.

Lebeau, I., Rees, G., \& Hughes, J. (2008). Language leader coursebook: Elementary. England: Pearson Education.

Lebeau, I., \& Rees, G. (2010). Language leader coursebook: Pre-intermediate. England: Pearson Education.

Lund, R. J. (1991). A comparison of second language listening and reading comprehension. Modern Language Journal, 75 (2), 196-204. MELAB Information Pack. Retrieved from www.cambridgemichigan.org/melab

Murphy, J. M. (1996). Integrating listening and reading instruction in EAP programs. English for Specific Purposes, 15 (2), 105-120.

Oxenden, C., \& Latham-Koenig, C., (2004). New English file: Elementary student"s book. Oxford: Oxford University Press.

Oxenden, C., Latham-Koenig, C., \& Seligson, P. (2005). New English file: Pre-intermediate student"s book. Oxford: Oxford University Press.

Oxenden, C., \& Latham-Koenig, C., (2006). New English file: Intermediate student"s book. Oxford: Oxford University Press.

Oxenden, C., \& Latham-Koenig, C., (2008). New English file: Upper-intermediate student"s book. Oxford: Oxford University Press.
Oxenden, C., \& Latham-Koenig, C., (2009). New English file: Beginner student"s book. Oxford: Oxford University Press. Oxenden, C., \& Latham-Koenig, C., (2010). New English file: Advanced student"s book. Oxford: Oxford University Press.

Powers. D. E. (1985). A survey of academic demands related to listening skills. TOEEL Res. Rep. No. 20. Princeton. NJ: Educational Testing Service.

Powers, D. E. (2013). Assessing English-language proficiency in all four language domains: Is it really necessary? $A$ Compendium of Studies, 2, (pp. 1.1-1.7) Princeton, NJ: Educational Testing Service.

PTE Academic Preparation Course Outline (2011), 2. Pearson Education. Retrieved from: http://pearsonpte.com/ wp-content/uploads/2014/07/Course-Outline.pdf

Redston, C., \& Cunningham, G. (2005). Face2face: Elementary student"s book. Cambridge: Cambridge University Press.

Redston, C., \& Cunningham, G. (2005). Face2face: Pre-intermediate student"s book. Cambridge: Cambridge University Press.

Redston, C., \& Cunningham, G. (2006). Face2face: Intermediate student"s book. Cambridge: Cambridge University Press.

Redston, C., \& Cunningham, G. (2007). Face2face: Upper-intermediate student"s book. Cambridge: Cambridge University Press.

Redston, C., \& Cunningham, G. (2009). Face2face: Starter student"s book. Cambridge: Cambridge University Press.

Richards, J. C. (1983). Listening comprehension: Approach, design, procedure. TESOL Quarterly, 17 (2), 219-240.

Richards, J. C. (1990). The language teaching matrix. Cambridge: Cambridge University Press.

Rost, M. (2011). Teaching and researching listening. Great Britain: Pearson Education.

Schroeders, U., Wilhelm, O., \& Bucholtz, N. (2010). Reading, listening, and viewing comprehension in English as a foreign language: One or more constructs?. Intelligence, 38 (6), 562-573. doi: 10.1016/j.intell.2010.09.003

Shiotsu, T. (2010). Components of $L 2$ reading: Linguistic and processing factors in the reading test performance of Japanese EFL learners. Cambridge: Cambridge University Press.

Song, M.Y. (2008). Do divisible subskills exist in second language (L2) comprehension? A structural equation modelling approach. Language Testing, 25(4), 435-464. doi:10.1177/0265532208094272.

Spies, T. G. (2011). Academic language proficiency development and its impact on reading comprehension: within and across languages (Doctoral dissertation). Texas A\&M University, USA. Retrieved from ProQuest (UMI Number: 3471240).

TOEFL Test Prep Planner (2012). Educational Testing Service. Retrieved from: https://www.ets.org/s/toefl/pdf/ toefl_student test prep planner.pdf

Unaldi, A. (2004). Construct validation of reading subskills of the Bogazici University English Proficiency Test (Doctoral Dissertation). Bogazici University, Turkey. Retrieved from Higher Education Council (Thesis number: 145737). 
Urquhart, A. H., \& Weir, C. J. (1998). Reading in a second language: Process, product and practice. New York: Longman.

Vandergrift, L. (2004). Listening to learn or learning to listen? Annual Review of Applied Linguistics, 24, 3-25. http://dx.doi.org/10.1017/S0267190504000017

Vandergrift, L. (2006). Second language listening: listening ability or language Proficiency? The Modern Language Journal, 90, 6-18.

Wagner, E. (2013). Assessing listening. In A. J. Kunan (Ed.). The companion to language assessment (47-63). Oxford: Wiley-Blackwell.

Weir, C. J. (1993). Understanding and developing language tests. UK: Phoenix.
Weir, C. J., \& Porter, D. (1994). The multi-divisible or unitary nature of reading: The language tester between Scylla and Charybdis. Reading in a Foreign Language, 107 (2), 1-19.

Weir, C.J., Huizhong, Y., \& Yan, J. (2000). An empirical investigation of the componentiality of $L 2$ reading in English for academic purposes. Cambridge: Cambridge University Press.

Weir, C. J. (2005). Language testing and validation: An evidence-based approach. Basingstoke: Palgrave Macmillan.

Weir, C. J., Hawkey, R., Green, A., Unaldi, A., \& Devi, S. (2009). The relationship between the academic reading construct as measured by IELTS and the reading experiences of students in their first year of study at a British university. IELTS Research Reports 9, 97-156. 
APPENDIX 1: List of RC \& LC Subskills Collected from Some Proficiency Tests

\begin{tabular}{lll}
\hline \multicolumn{1}{c}{ RC sub-skills } & \multicolumn{1}{c}{ LC sub-skills } \\
\hline & \multicolumn{1}{c}{ TOEFL IBT } \\
\hline 1. Understanding main ideas & 1. Understanding main idea and general topic \\
2. Understanding facts and details including & 2. Understanding facts and details \\
negative facts & 3. Understanding purpose of conversation or \\
3. Making inferences about the content & & lecture (relations between ideas) \\
4. Identifying purpose of the author about details & 4. Understanding the function of the message \\
in content (relations between ideas) & 5. Understanding a speaker's attitude \\
5. Understanding a meaning of unknown & 6. Understanding organization of information \\
vocabulary & 7. Connecting the content by identifying \\
6. Understanding pronoun reference & & comparisons, cause and effect, or \\
7. Sentence paraphrasing & 8. Making inferences \\
8. Inserting a sentence in an appropriate gap & & \\
9. Summarizing by distinguishing major and & \\
minor ideas and cause-effect, compare- & \\
contrast relationships, arguments etc. &
\end{tabular}

IELTS Academic

1. Understanding main idea and general topic
2. Understanding specific information and
finding details
3. Recognizing opinions and ideas and writer's
claims
4. Summarizing details or main ideas in
table/note/flow/chart
5. Understanding detailed description and relating
it to information given in a diagram
6. Completing a sentence by finding detailed
information
7. Finding factual details (specific information) by
short answer questions

1. Understanding main idea

2. Listening detailed information

3. Recognizing how facts are connected to each other

4. Understanding descriptions, explanations, directions, and relating them to plan/map/diagram

5. Summarizing information

6. Completing a sentence by identifying important information and relationship between ideas/facts/events/cause/effect

7. Listening for facts and answering short answer questions

\begin{tabular}{ll}
\hline & Cambridge Englis \\
\hline 1. Understanding general meaning & \\
2. Understanding details and finding specific \\
information \\
3. Understanding opinions and attitudes \\
4. Understanding the structure and following a \\
development of a text
\end{tabular}

\section{Cambridge English: Advanced (CAE)}

1. Understanding main idea

2. Understanding details and specific information

3. Understanding opinion, purpose and attitude

4. Understanding implications

5. Understanding structure and development of a text
1. Listening for main points

2. Listening for details

3. Understanding feeling, attitude, opinion, function, course of action

4. Interpreting context

\section{Cambridge English Proficiency (CPE)}

1. Understanding main idea and global points

2. Understanding details

3. Understanding cohesion, coherence and text
1. Listening for gist and topic

2. Listening for specific information and details 3 . Inferencing 
structure

4. Awareness and control of grammar with focus on vocabulary

5. Understanding and using idioms, collocations, fixed phrases, complementation, phrasal verbs, semantic precision

6. Understanding opinion and attitude
4. Understanding addressee, feeling, attitude, opinion, function, purpose and interpreting context

Pearson P
1. Identifying the topic
2. Identifying supporting points or examples
Identifying a summary
3. Identifying words and phrases appropriate to the
context
4. Identifying a writer's purpose, tone,
technique and attitude
5. Identifying the relationships between
6. Understanding academic vocabulary
7. Understanding the difference between
connotation and denotation
9. Inferring the meaning of unfamiliar words
9. Comprehending explicit and implicit

\section{E Academic}

1. Identifying the topic

2. Summarizing the main idea

3. Identifying supporting points or examples

4. Understanding academic vocabulary

5. Inferring the meaning of unfamiliar words

6. Identifying words and phrases appropriate to the context

7. Comprehending explicit and implicit information

8. Comprehending concrete and abstract information

9. Classifying and categorizing information

10. Following an oral sequencing of information

11. Critically evaluating information presented

12. Forming a conclusion from what a speaker says

13. Predicting how a speaker may continue

14. Identifying errors in a transcription

15. Identifying a speaker's purpose, tone and attitude

16. Identifying the framework used to convey information (e.g., generalization, conclusion, cause and effect)

17. Inferring the context, purpose or tone

18. Comprehending variations in tone, speed, accent

\begin{tabular}{|c|c|}
\hline \multicolumn{2}{|c|}{ Canadian Academic English Language Assessment (CAEL) } \\
\hline 1. Identifying main idea & 1. Identifying main ideas \\
\hline 2. Extracting specific information & 2. Completing charts or diagrams \\
\hline 3. Understanding vocabulary in context & 3. Sequencing information \\
\hline 4. Classifying information & 4. Taking notes \\
\hline 5. Following a logical or chronological & 5. Filling in the blanks \\
\hline sequence of events & 6. Recording specific information \\
\hline \multicolumn{2}{|c|}{ Michigan English Language Assessment Battery (MELAB) } \\
\hline 1. Understanding main idea & 1. Understanding main idea \\
\hline 2. Identifying speaker's purpose & speaker's purpose \\
\hline 3. Synthesizing ideas from different parts of the text & 3. Synthesizing ideas from different parts of the \\
\hline $\begin{array}{l}\text { 4. Identifying supporting detail } \\
\text { 5. Understanding vocabulary }\end{array}$ & 4. Identifying supporting detail \\
\hline 6. Synthesizing details & 5. Understanding vocabulary \\
\hline 7. Recognizing restatement & 6. Synthesizing details \\
\hline 8. Understanding rhetorical function & 7. Recognizing restatement \\
\hline 9. Making an inference & 8. Understanding rhetorical function \\
\hline 10. Inferring supporting detail & 9. Making an inference \\
\hline & 10. Inferring supporting detail \\
\hline
\end{tabular}





\section{+MELAB Grammar, Cloze and Vocabulary section}

Grammar sub-skill: testing different grammatical features

Cloze sub-skills:

Selecting the best word (or phrase) to restore the intended meaning of a chunk of text;

Identifying the correct grammatical form of a word (or phrase) for the blank; Identifying the most appropriate content word (or phrase) for the blank

Vocabulary sub-skill: Measuring vocabulary size and certain in-depth aspects of vocabulary knowledge

Examination for the Certificate of Competency in English (ECCE)

1. Understanding main idea

2. Understanding author's opinion

3. Understanding the relationship between ideas

4. Comparing/contrasting features of one or more texts

5. Understanding explicitly stated ideas (detail) from one or more texts

6. Understanding vocabulary in context

7. Identifying referents

8. Drawing an inference/conclusion from one or more texts

9. Understanding rhetorical function
1. Understanding main idea

2. Identifying speaker's mood/attitude/opinion

3. Synthesizing information

4. Understanding explicitly stated ideas (detail)

5. Understanding vocabulary in context

6. Drawing an inference/conclusion

7. Understanding rhetorical function

8. Making predictions

\section{+ECCE Grammar and Vocabulary section}

Grammar sub-skills: measure grammatical features observed in written American English. Vocabulary sub-skills: measure vocabulary size and tap certain in-depth aspects of vocabulary knowledge.

\section{APPENDIX 2: List of Analyzed EFL/ESL Textbooks}

\begin{tabular}{|c|c|c|c|c|}
\hline & Author(s) & Title of the Book & $\begin{array}{l}\text { Language } \\
\text { Proficiency } \\
\text { Level }\end{array}$ & $\begin{array}{c}\text { Date of } \\
\text { Publication }\end{array}$ \\
\hline 1. & Oxenden, C., \& Latham-Koenig, C. & New English File & Beginner & 2009 \\
\hline 2. & Oxenden, C., \& Latham-Koenig, C. & New English File & Elementary & 2004 \\
\hline 3. & $\begin{array}{l}\text { Oxenden, C., Latham-Koenig, C., \& } \\
\text { Seligson, P. }\end{array}$ & New English File & $\begin{array}{l}\text { Pre- } \\
\text { intermediate }\end{array}$ & 2005 \\
\hline 4. & Oxenden, C., \& Latham-Koenig, C. & New English File & Intermediate & 2006 \\
\hline 5. & Oxenden, C., \& Latham-Koenig, C. & New English File & $\begin{array}{l}\text { Upper- } \\
\text { intermediate }\end{array}$ & 2008 \\
\hline 6. & Oxenden, C., \& Latham-Koenig, C. & New English File & Advanced & 2010 \\
\hline 7. & Redston, C., \& Cunningham, G. & Face2Face & $\begin{array}{c}\text { Starter } \\
\text { (Beginner) }\end{array}$ & 2009 \\
\hline 8. & Redston, C., \& Cunningham, G. & Face2Face & Elementary & 2005 \\
\hline 9. & Redston, C., \& Cunningham, G. & Face2Face & $\begin{array}{c}\text { Pre- } \\
\text { intermediate }\end{array}$ & 2005 \\
\hline 10. & Redston, C., \& Cunningham, G. & Face2Face & Intermediate & 2006 \\
\hline 11. & Redston, C., \& Cunningham, G. & Face2Face & $\begin{array}{l}\text { Upper- } \\
\text { intermediate }\end{array}$ & 2007 \\
\hline 12. & $\begin{array}{l}\text { Cunningham, G., Bell, J., \& Redston, } \\
\text { C. }\end{array}$ & Face2Face & Advanced & 2009 \\
\hline 13. & $\begin{array}{l}\text { Kay, S., Jones, V., Gomm, H., } \\
\text { Seymour, D., Brown, C., et al }\end{array}$ & New Inside Out & Beginner & 2010 \\
\hline 14. & Kay, S., \& Jones, V. & New Inside Out & Elementary & 2007 \\
\hline
\end{tabular}


15. Kay, S., \& Jones, V.

16. Kay, S., \& Jones, V.

17. Kay, S., \& Jones, V.

18. Kay, S., Jones, V., Gomm, H., Maggs, P., \& Dawson, C.

19. Dellar, H., \& Walkley, A.

20. Dellar, H., \& Walkley, A.

21. Dellar, H., \& Walkley, A.

22. Dellar, H., \& Walkley, A.

23. Dellar, H., \& Walkley, A.

24. Lebeau, I., Rees, G., \& Hughes, J.

25. Lebeau, I., \& Rees, G.

26. Cotton, D., Falvey, D., \& Kent, S.

27. Cotton, D., Falvey, D., \& Kent, S.

28. Cotton, D., Falvey, D., Kent, S., Lebeau, I., \& Rees, G.

$\begin{array}{ccc}\text { New Inside Out } & \text { Pre- } & 2008 \\ \text { New Inside Out } & \begin{array}{c}\text { intermediate } \\ \text { Intermediate }\end{array} & 2009 \\ \text { New Inside Out } & \text { Upper- } & 2009 \\ & \text { intermediate } & \\ \text { New Inside Out } & \text { Advanced } & 2010 \\ \text { Outcomes } & \text { Elementary } & 2011 \\ \text { Outcomes } & \text { Pre- } & 2010 \\ \text { Outcomes } & \text { intermediate } & \\ \text { Outcomes } & \text { Intermediate } & 2010 \\ & \text { Upper- } & 2010 \\ \text { Outcomes } & \text { intermediate } & \\ \text { Language Leader } & \text { Advanced } & 2012 \\ \text { Language Leader } & \text { Elementary } & 2008 \\ & \text { Pre- } & 2010 \\ \text { Language Leader } & \text { intermediate } & \\ \text { Language Leader } & \text { Intermediate } & 2008 \\ & \text { Upper- } & 2008 \\ \text { Language Leader } & \text { intermediate } & \\ & \text { Advanced } & 2010\end{array}$


APPENDIX 3: List of ELT RC \& LC Tasks from Textbooks (New English File, Face2Face, New Inside Out, Outcomes and Language Leader)

\begin{tabular}{|c|c|c|c|c|c|}
\hline & $\begin{array}{c}\text { New English File } \\
\text { Advanced }\end{array}$ & $\begin{array}{l}\text { Face2face } \\
\text { Advanced }\end{array}$ & New Inside Out & $\begin{array}{l}\text { Outcomes } \\
\text { Advanced }\end{array}$ & $\begin{array}{c}\text { Language Leader } \\
\text { Advanced }\end{array}$ \\
\hline $\begin{array}{c}\text { Reading } \\
\text { tasks }\end{array}$ & $\begin{array}{l}\text { Understanding main idea } \\
\text { Understanding facts and details } \\
\text { Understanding writer's attitude } \\
\text { and purpose } \\
\text { Inferring information from a } \\
\text { context } \\
\text { Inferring a meaning of } \\
\text { unknown word from a context } \\
\text { Matching headings to } \\
\text { paragraphs } \\
\text { Noticing discourse markers in } \\
\text { reading and highlighting them } \\
\text { Matching words from reading } \\
\text { to their definitions } \\
\text { Finding synonyms to words } \\
\text { from reading } \\
\text { Paraphrasing a text } \\
\text { Guessing the content of } \\
\text { reading by pictures and } \\
\text { introduction } \\
\text { Recognizing/using grammar } \\
\text { points or grammar in context } \\
\text { Discussing reading text } \\
\text { questions alone/in pairs }\end{array}$ & $\begin{array}{l}\text { Understanding main idea } \\
\text { Understanding facts and details } \\
\text { Understanding writer's attitude } \\
\text { and purpose } \\
\text { Inferring information from a } \\
\text { context } \\
\text { Inferring a meaning of } \\
\text { unknown word from a context } \\
\text { Completing sentences with } \\
\text { missing words/phrases in } \\
\text { context } \\
\text { Finding synonyms/antonyms of } \\
\text { words/phrases in context } \\
\text { Listening and repeating phrases } \\
\text { Matching sentence beginnings } \\
\text { to their endings } \\
\text { Matching headings to } \\
\text { paragraphs } \\
\text { Matching statements to pictures } \\
\text { Recognizing functions of } \\
\text { words/phrases/ statements } \\
\text { Recognizing/using correct } \\
\text { grammar points or grammar in } \\
\text { context } \\
\text { Paraphrasing sentences } \\
\text { Discussing reading text } \\
\text { questions alone/in pairs }\end{array}$ & $\begin{array}{l}\text { Understanding main idea } \\
\text { Understanding facts and } \\
\text { details } \\
\text { Understanding writer‘s attitude } \\
\text { and purpose } \\
\text { Inferring information from a } \\
\text { context } \\
\text { Inferring meaning of unknown } \\
\text { word from a context } \\
\text { Completing sentences with } \\
\text { missing words/phrases in } \\
\text { context } \\
\text { Matching words from a text } \\
\text { with their collocations } \\
\text { Matching headings to } \\
\text { paragraphs } \\
\text { Summarizing information } \\
\text { Inserting missing sentences in } \\
\text { the article } \\
\text { Paraphrasing a text } \\
\text { Recognizing/using grammar } \\
\text { points or grammar in context } \\
\text { Discussing reading text } \\
\text { questions alone/in pairs }\end{array}$ & $\begin{array}{l}\text { Understanding main idea } \\
\text { Understanding facts and } \\
\text { details } \\
\text { Understanding writer's } \\
\text { attitude and purpose } \\
\text { Inferring information from a } \\
\text { context } \\
\text { Summarizing information } \\
\text { Completing sentences with } \\
\text { missing words/phrases in } \\
\text { context } \\
\text { Retelling history with new } \\
\text { vocabulary } \\
\text { Discussing reading text } \\
\text { questions alone/in pairs }\end{array}$ & $\begin{array}{l}\text { Understanding main idea } \\
\text { Understanding facts and } \\
\text { details } \\
\text { Inferring information from } \\
\text { context } \\
\text { Inferring meaning of } \\
\text { unknown word from a context } \\
\text { Understanding writer's } \\
\text { attitude and purpose } \\
\text { Summarizing information } \\
\text { Recognizing/using grammar } \\
\text { points or grammar in context } \\
\text { Identifying reference words in } \\
\text { a text } \\
\text { Matching headings to } \\
\text { paragraphs } \\
\text { Completing sentences with } \\
\text { missing words/phrases in } \\
\text { context } \\
\text { Discussing reading text } \\
\text { questions alone/in pairs }\end{array}$ \\
\hline $\begin{array}{l}\text { Listening } \\
\text { tasks }\end{array}$ & $\begin{array}{l}\text { Understanding main idea } \\
\text { Understanding facts and details } \\
\text { Understanding speaker's } \\
\text { attitude and purpose }\end{array}$ & $\begin{array}{l}\text { Understanding main idea } \\
\text { Understanding facts and details } \\
\text { Inferring information from a } \\
\text { context }\end{array}$ & $\begin{array}{l}\text { Understanding main idea } \\
\text { Understanding facts and } \\
\text { details } \\
\text { Understanding writer's attitude }\end{array}$ & $\begin{array}{l}\text { Understanding main idea } \\
\text { Understanding facts and } \\
\text { details } \\
\text { Understanding speaker's }\end{array}$ & $\begin{array}{l}\text { Understanding main idea } \\
\text { Understanding facts and } \\
\text { details } \\
\text { Inferring information from a }\end{array}$ \\
\hline
\end{tabular}




\begin{tabular}{|c|c|c|c|c|c|}
\hline & $\begin{array}{l}\text { Inferring information from a } \\
\text { context } \\
\text { Inferring a meaning of } \\
\text { unknown word from a context } \\
\text { Summarizing information } \\
\text { Recognizing functions of } \\
\text { phrases in context } \\
\text { Listening and noting key } \\
\text { words } \\
\text { Listening and completing } \\
\text { sentences/extracts with } \\
\text { missing words/phrases } \\
\text { Listening and completing a } \\
\text { table } \\
\text { Discussing } \\
\text { listening text questions } \\
\text { alone/in pairs }\end{array}$ & $\begin{array}{l}\text { Understanding speaker's } \\
\text { attitude and purpose } \\
\text { Inferring a meaning of } \\
\text { unknown word from context } \\
\text { Listening and completing } \\
\text { sentences/extracts with missing } \\
\text { words/phrases } \\
\text { Listening and repeating } \\
\text { sentences } \\
\text { Recognizing functions of } \\
\text { words/phrases/statements } \\
\text { Predicting the end of history } \\
\text { Matching statements/extracts to } \\
\text { pictures } \\
\text { Summarizing information } \\
\text { Taking notes under headings } \\
\text { Discussing } \\
\text { listening text questions } \\
\text { alone/in pairs }\end{array}$ & $\begin{array}{l}\text { and purpose } \\
\text { Inferring information from a } \\
\text { context } \\
\text { Inferring a meaning of } \\
\text { unknown word from a context } \\
\text { Listening and completing } \\
\text { sentences/extract with missing } \\
\text { words/phrases } \\
\text { Listening and matching } \\
\text { situations to pictures } \\
\text { Listening and matching } \\
\text { statements/extracts to people } \\
\text { Listening and ordering pictures } \\
\text { according to the recording } \\
\text { Perceiving and discriminating } \\
\text { individual sounds } \\
\text { Predicting a speakers' message } \\
\text { Discussing } \\
\text { listening text questions } \\
\text { alone/in pairs }\end{array}$ & $\begin{array}{l}\text { attitude and purpose } \\
\text { Inferring information from a } \\
\text { context } \\
\text { Taking notes } \\
\text { Summarizing information } \\
\text { Listening and completing } \\
\text { sentences with missing } \\
\text { words/phrases } \\
\text { Listening and matching } \\
\text { statements/extracts to pictures } \\
\text { Listening and writing down } \\
\text { unfamiliar words/expressions } \\
\text { Listening and repeating } \\
\text { expressions } \\
\text { Recognizing functions of } \\
\text { words/phrases/statements } \\
\text { Practicing listening } \\
\text { conversations/topic in } \\
\text { speaking tasks with peers } \\
\text { Predicting the end of story } \\
\text { Discussing } \\
\text { listening text questions } \\
\text { alone/in pairs }\end{array}$ & $\begin{array}{l}\text { context } \\
\text { Understanding speaker's } \\
\text { attitude and purpose } \\
\text { Summarizing information } \\
\text { Recognizing function of } \\
\text { phrases in context } \\
\text { Predicting a speaker's } \\
\text { message } \\
\text { Listening and completing } \\
\text { sentences/ extracts with } \\
\text { missing words/ phrases } \\
\text { Listening and paraphrasing } \\
\text { sentences } \\
\text { Listening and ordering } \\
\text { sentences according to } \\
\text { recording } \\
\text { Taking notes under headings } \\
\text { Discussing } \\
\text { listening text questions } \\
\text { alone/in pairs }\end{array}$ \\
\hline & Upper-intermediate & Upper-intermediate & Upper-intermediate & Upper-intermediate & Upper-intermediate \\
\hline $\begin{array}{l}\text { Reading } \\
\text { tasks }\end{array}$ & $\begin{array}{l}\text { Understanding main idea } \\
\text { Understanding facts and details } \\
\text { Understanding writer's attitude } \\
\text { and purpose } \\
\text { Inferring information from a } \\
\text { context } \\
\text { Inferring a meaning of } \\
\text { unknown word from a context } \\
\text { Inferring information from } \\
\text { passage with pictures } \\
\text { Summarizing information } \\
\text { Matching words from reading } \\
\text { to their definitions } \\
\text { Completing sentences using } \\
\text { words/phrases }\end{array}$ & $\begin{array}{l}\text { Understanding main idea } \\
\text { Understanding facts and details } \\
\text { Inferring information from a } \\
\text { context } \\
\text { Understanding writer's attitude } \\
\text { and purpose } \\
\text { Inferring a meaning of } \\
\text { unknown word from a context } \\
\text { Recognizing/using correct } \\
\text { grammar points or grammar in } \\
\text { context } \\
\text { Recognizing functions of } \\
\text { words/phrases/statements } \\
\text { Matching headings to } \\
\text { paragraphs }\end{array}$ & $\begin{array}{l}\text { Understanding main idea } \\
\text { Understanding facts and } \\
\text { details } \\
\text { Understanding writer‘s attitude } \\
\text { and purpose } \\
\text { Inferring information from a } \\
\text { context } \\
\text { Inferring a meaning of } \\
\text { unknown word from a context } \\
\text { Summarizing information } \\
\text { Completing sentences with } \\
\text { missing words/phrases in } \\
\text { context } \\
\text { Recognizing/using correct } \\
\text { grammar points or grammar in }\end{array}$ & $\begin{array}{l}\text { Understanding main idea } \\
\text { Understanding facts and } \\
\text { details } \\
\text { Understanding writer's } \\
\text { attitude and purpose } \\
\text { Inferring information from a } \\
\text { context } \\
\text { Summarizing information } \\
\text { Completing sentences with } \\
\text { missing words/phrases in } \\
\text { context } \\
\text { Recognizing/using correct } \\
\text { grammar points or grammar in } \\
\text { context } \\
\text { Reading and replacing }\end{array}$ & $\begin{array}{l}\text { Understanding main idea } \\
\text { Understanding facts and } \\
\text { details } \\
\text { Inferring information from a } \\
\text { context } \\
\text { Inferring a meaning of } \\
\text { unknown word from a context } \\
\text { Matching statements to } \\
\text { pictures } \\
\text { Matching headings to } \\
\text { paragraphs } \\
\text { Matching titles to paragraphs } \\
\text { Numbering statements from a } \\
\text { text in a chronological order } \\
\text { Completing sentences with }\end{array}$ \\
\hline
\end{tabular}




\begin{tabular}{|c|c|c|c|c|c|}
\hline & $\begin{array}{l}\text { Matching statements to } \\
\text { pictures } \\
\text { Recognizing/using grammar } \\
\text { points or grammar in context } \\
\text { Discussing reading text } \\
\text { questions alone/in pairs }\end{array}$ & $\begin{array}{l}\text { Matching headings to } \\
\text { statements } \\
\text { Identifying reference words in a } \\
\text { text } \\
\text { Discussing reading text } \\
\text { questions alone/in pairs }\end{array}$ & $\begin{array}{l}\text { context } \\
\text { Matching heading to } \\
\text { paragraphs } \\
\text { Discussing reading text } \\
\text { questions alone/in pairs }\end{array}$ & $\begin{array}{l}\text { selected words with synonyms } \\
\text { Reading and completing } \\
\text { collocations } \\
\text { Matching headings to } \\
\text { paragraphs } \\
\text { Discussing reading text } \\
\text { questions alone/in pairs }\end{array}$ & $\begin{array}{l}\text { missing words/phrases in } \\
\text { context } \\
\text { Finding synonyms of given } \\
\text { words/phrases from a text } \\
\text { Recognizing function of } \\
\text { phrases in context } \\
\text { Matching definition of words } \\
\text { from in a context } \\
\text { Summarizing information } \\
\text { Discussing reading text } \\
\text { questions alone/in pairs }\end{array}$ \\
\hline $\begin{array}{l}\text { Listening } \\
\text { tasks }\end{array}$ & $\begin{array}{l}\text { Understanding main idea } \\
\text { Understanding facts and details } \\
\text { Understanding speaker's } \\
\text { attitude and purpose } \\
\text { Inferring information from a } \\
\text { context } \\
\text { Inferring a meaning of } \\
\text { unknown word from a context } \\
\text { Predicting/guessing speaker's } \\
\text { message } \\
\text { Listening and completing } \\
\text { sentences with missing } \\
\text { words/phrases } \\
\text { Understanding unknown word } \\
\text { in context } \\
\text { Listening and matching } \\
\text { statements to pictures } \\
\text { Understanding accent of } \\
\text { speakers and matching them to } \\
\text { their countries } \\
\text { Listening and completing a } \\
\text { table } \\
\text { Listening and completing notes } \\
\text { Discussing } \\
\text { listening text questions } \\
\text { alone/in pairs }\end{array}$ & $\begin{array}{l}\text { Understanding main idea } \\
\text { Understanding facts and details } \\
\text { Inferring information from a } \\
\text { context } \\
\text { Understanding speaker‘s } \\
\text { attitude and purpose } \\
\text { Listening and completing } \\
\text { sentences/extracts with missing } \\
\text { words/phrases } \\
\text { Predicting a speaker‘s message } \\
\text { Recognizing functions of } \\
\text { words/phrases/statements } \\
\text { Listening and writing sentences } \\
\text { Perceiving and discriminating } \\
\text { individual sounds } \\
\text { Discussing } \\
\text { listening text questions } \\
\text { alone/in pairs }\end{array}$ & $\begin{array}{l}\text { Understanding main idea } \\
\text { Understanding facts and } \\
\text { details } \\
\text { Understanding speaker's } \\
\text { attitude and purpose } \\
\text { Inferring information from a } \\
\text { context } \\
\text { Matching statements/extracts } \\
\text { to pictures } \\
\text { Listening and completing a } \\
\text { table } \\
\text { Listening and completing } \\
\text { sentences/extracts with } \\
\text { missing words/phrases } \\
\text { Recognizing functions of } \\
\text { words/phrases/statements } \\
\text { Discussing } \\
\text { listening text questions } \\
\text { alone/in pairs }\end{array}$ & $\begin{array}{l}\text { Understanding main idea } \\
\text { Understanding facts and } \\
\text { details } \\
\text { Understanding speaker's } \\
\text { attitude and purpose } \\
\text { Inferring information from a } \\
\text { context } \\
\text { Perceiving and discriminating } \\
\text { individual sounds } \\
\text { Listening and writing down } \\
\text { unfamiliar words/expressions } \\
\text { Listening and completing } \\
\text { sentences with missing } \\
\text { words/phrases } \\
\text { Listening and taking notes } \\
\text { Listen and speaking using } \\
\text { sentence frames from } \\
\text { listening passage } \\
\text { Listening and matching } \\
\text { statements/extracts to pictures } \\
\text { Predicting the end of story } \\
\text { Listening and completing a } \\
\text { table } \\
\text { Recognizing functions of } \\
\text { words/phrases/statements } \\
\text { Discussing } \\
\text { listening text questions }\end{array}$ & $\begin{array}{l}\text { Understanding main idea } \\
\text { Understanding facts and } \\
\text { details } \\
\text { Inferring information from } \\
\text { context } \\
\text { Listening and competing } \\
\text { sentences/ extracts with } \\
\text { missing words/phrases } \\
\text { Recognizing function of } \\
\text { phrases in context } \\
\text { Taking notes about main } \\
\text { points } \\
\text { Listening and ordering } \\
\text { sentences according to } \\
\text { recording } \\
\text { Listening and repeating } \\
\text { expressions } \\
\text { Listening and completing a } \\
\text { table } \\
\text { Discussing } \\
\text { listening text questions } \\
\text { alone/in pairs }\end{array}$ \\
\hline
\end{tabular}




\begin{tabular}{|c|c|c|c|c|c|}
\hline & & & & alone/in pairs & \\
\hline & Intermediate & Intermediate & Intermediate & Intermediate & Intermediate \\
\hline $\begin{array}{l}\text { Reading } \\
\text { tasks }\end{array}$ & $\begin{array}{l}\text { Understanding main idea } \\
\text { Understanding facts and details } \\
\text { Understanding writer's attitude } \\
\text { and purpose } \\
\text { Inferring information from a } \\
\text { context } \\
\text { Inferring a meaning of } \\
\text { unknown word from a context } \\
\text { Matching words from reading } \\
\text { to their definitions } \\
\text { Recognizing/using grammar } \\
\text { points or grammar in context } \\
\text { Inserting a sentence into gaps } \\
\text { in paragraphs } \\
\text { Matching words from reading } \\
\text { to their definitions } \\
\text { Completing sentences with } \\
\text { words/phrases in a context } \\
\text { Reading and completing a } \\
\text { table } \\
\text { Numbering paragraphs in the } \\
\text { correct order } \\
\text { Discussing reading text } \\
\text { questions alone/in pairs }\end{array}$ & $\begin{array}{l}\text { Understanding main idea } \\
\text { Understanding facts and details } \\
\text { Inferring information from a } \\
\text { context } \\
\text { Inferring a meaning of } \\
\text { unknown word from context } \\
\text { Matching headings to } \\
\text { paragraphs } \\
\text { Recognizing/using correct } \\
\text { grammar points or grammar in } \\
\text { context } \\
\text { Completing sentences with } \\
\text { missing words/phrases in } \\
\text { context } \\
\text { Reading and completing a table } \\
\text { Discussing reading text } \\
\text { questions alone/in pairs }\end{array}$ & $\begin{array}{l}\text { Understanding main idea } \\
\text { Understanding facts and } \\
\text { details } \\
\text { Inferring information from a } \\
\text { context } \\
\text { Completing sentences with } \\
\text { missing words/phrases in } \\
\text { context } \\
\text { Matching statements to } \\
\text { pictures } \\
\text { Matching headings to } \\
\text { paragraphs } \\
\text { Discussing reading text } \\
\text { questions alone/in pairs }\end{array}$ & $\begin{array}{l}\text { Understanding main idea } \\
\text { Understanding facts and } \\
\text { details } \\
\text { Understanding writer's } \\
\text { attitude and purpose } \\
\text { Inferring information from a } \\
\text { context } \\
\text { Reading and finding correct } \\
\text { collocations } \\
\text { Completing sentence with } \\
\text { correct words/phrases in } \\
\text { context } \\
\text { Completing dialogues with } \\
\text { correct word order } \\
\text { Guessing/predicting the end } \\
\text { of history } \\
\text { Matching passages to } \\
\text { headings } \\
\text { Discussing reading text } \\
\text { questions alone/in pairs }\end{array}$ & $\begin{array}{l}\text { Understanding main idea } \\
\text { Understanding facts and } \\
\text { details } \\
\text { Inferring information from a } \\
\text { context } \\
\text { Summarizing information } \\
\text { Matching statements/headings } \\
\text { to paragraphs } \\
\text { Inferring meaning of } \\
\text { unfamiliar word from a } \\
\text { context } \\
\text { Completing sentences with } \\
\text { missing words/phrases in } \\
\text { context } \\
\text { Recognizing/using grammar } \\
\text { points or grammar in context } \\
\text { Identifying reference words in } \\
\text { a text } \\
\text { Reading and completing a } \\
\text { table } \\
\text { Discussing reading text } \\
\text { questions alone/in pairs }\end{array}$ \\
\hline $\begin{array}{l}\text { Listening } \\
\text { tasks }\end{array}$ & $\begin{array}{l}\text { Understanding main idea } \\
\text { Understanding facts and details } \\
\text { Understanding speaker's } \\
\text { attitude and purpose } \\
\text { Inferring information from a } \\
\text { context } \\
\text { Listening and completing } \\
\text { sentences with words/ phrases } \\
\text { Listening and repeating the } \\
\text { words/phrases /sentences } \\
\text { Matching words from listening } \\
\text { to their definitions }\end{array}$ & $\begin{array}{l}\text { Understanding main idea } \\
\text { Understanding facts and details } \\
\text { Inferring information from a } \\
\text { context } \\
\text { Understanding speaker's } \\
\text { attitude and purpose } \\
\text { Perceiving and discriminating } \\
\text { individual sounds } \\
\text { Predicting a speaker's message } \\
\text { Listening and completing } \\
\text { sentences/extracts with missing } \\
\text { words/phrases }\end{array}$ & $\begin{array}{l}\text { Understanding main idea } \\
\text { Understanding facts and } \\
\text { details } \\
\text { Inferring information from a } \\
\text { context } \\
\text { Listening and completing } \\
\text { sentences/extract with missing } \\
\text { words/phrases } \\
\text { Listening and repeating } \\
\text { phrases } \\
\text { Matching headings to extracts } \\
\text { from recording }\end{array}$ & $\begin{array}{l}\text { Understanding main idea } \\
\text { Understanding facts and } \\
\text { details } \\
\text { Understanding speaker‘s } \\
\text { attitude and purpose } \\
\text { Inferring information from a } \\
\text { context } \\
\text { Perceiving and discriminating } \\
\text { individual sounds } \\
\text { Listening and completing } \\
\text { sentences with missing } \\
\text { words/phrases }\end{array}$ & $\begin{array}{l}\text { Understanding main idea } \\
\text { Understanding facts and } \\
\text { details } \\
\text { Inferring information from a } \\
\text { context } \\
\text { Understanding speaker's } \\
\text { attitude and purpose } \\
\text { Summarizing information } \\
\text { Listening and competing } \\
\text { sentences/extracts with } \\
\text { missing words/phrases } \\
\text { Perceiving and discriminating }\end{array}$ \\
\hline
\end{tabular}




\begin{tabular}{|c|c|c|c|c|c|}
\hline & $\begin{array}{l}\text { Listening and completing a } \\
\text { table } \\
\text { Taking notes } \\
\text { Matching statements/extracts } \\
\text { to pictures } \\
\text { Discussing } \\
\text { listening text questions } \\
\text { alone/in pairs }\end{array}$ & $\begin{array}{l}\text { Matching statements/extracts to } \\
\text { pictures } \\
\text { Discussing } \\
\text { listening text questions } \\
\text { alone/in pairs }\end{array}$ & $\begin{array}{l}\text { Listening and ordering pictures } \\
\text { according to the recording } \\
\text { Discussing } \\
\text { listening text questions } \\
\text { alone/in pairs }\end{array}$ & $\begin{array}{l}\text { Listening and matching } \\
\text { collocations } \\
\text { Listening and completing } \\
\text { sentences by replacing } \\
\text { collocations with synonyms } \\
\text { Listening and completing a } \\
\text { table } \\
\text { Listen and role playing the } \\
\text { conversations } \\
\text { Developing a conversation by } \\
\text { using phrases from listening } \\
\text { Discussing } \\
\text { listening text questions } \\
\text { alone/in pairs }\end{array}$ & $\begin{array}{l}\text { individual sounds } \\
\text { Recognizing function of } \\
\text { phrases in context } \\
\text { Taking notes by filling a gap } \\
\text { in extracts } \\
\text { Listening and completing a } \\
\text { table } \\
\text { Discussing } \\
\text { listening text questions } \\
\text { alone/in pairs }\end{array}$ \\
\hline & Pre-intermediate & Pre-intermediate & Pre-intermediate & Pre-intermediate & Pre-intermediate \\
\hline $\begin{array}{l}\text { Reading } \\
\text { tasks }\end{array}$ & $\begin{array}{l}\text { Understanding facts and details } \\
\text { Inferring a meaning of } \\
\text { unknown word in a context } \\
\text { Matching definitions in gaps } \\
\text { according to descriptions } \\
\text { Finding synonyms and } \\
\text { antonyms of words } \\
\text { Underlining unknown words } \\
\text { and expressions and check } \\
\text { with dictionary } \\
\text { Reading a text and practicing } \\
\text { grammar points } \\
\text { Reading for facts and details } \\
\text { Matching headings with } \\
\text { paragraphs } \\
\text { Inferring reader's attitude and } \\
\text { purpose } \\
\text { Reading a letter or a paragraph } \\
\text { and matching with pictures } \\
\text { Answering negative answer } \\
\text { questions } \\
\text { Numbering sentences in } \\
\text { chronological order } \\
\text { Placing paragraphs in correct }\end{array}$ & $\begin{array}{l}\text { Understanding main idea } \\
\text { Understanding facts and details } \\
\text { Inferring information from a } \\
\text { context } \\
\text { Inferring a meaning of } \\
\text { unknown word from a context } \\
\text { Matching headings to } \\
\text { paragraphs } \\
\text { Matching statements to pictures } \\
\text { Recognizing/using correct } \\
\text { grammar points or grammar in } \\
\text { context } \\
\text { Discussing reading text } \\
\text { questions alone/in pairs }\end{array}$ & $\begin{array}{l}\text { Understanding main idea } \\
\text { Understanding facts and } \\
\text { details } \\
\text { Matching headings to } \\
\text { paragraphs } \\
\text { Completing sentences with } \\
\text { missing words/phrases in } \\
\text { context } \\
\text { Matching statements } \\
\text { /paragraphs to pictures } \\
\text { Discussing reading text } \\
\text { questions alone/in pairs }\end{array}$ & $\begin{array}{l}\text { Understanding main idea } \\
\text { Understanding facts and } \\
\text { details } \\
\text { Understanding writer's } \\
\text { attitude and purpose } \\
\text { Inferring information from a } \\
\text { context } \\
\text { Completing sentences with } \\
\text { missing words/phrases in } \\
\text { context } \\
\text { Matching selected words from } \\
\text { text to their definitions } \\
\text { Matching passages to pictures } \\
\text { Matching passages to } \\
\text { headings } \\
\text { Discussing reading text } \\
\text { questions alone/in pairs }\end{array}$ & $\begin{array}{l}\text { Understanding main idea } \\
\text { Understanding facts and } \\
\text { details } \\
\text { Inferring information from a } \\
\text { context } \\
\text { Summarizing information } \\
\text { Inferring meaning of } \\
\text { unfamiliar word from a } \\
\text { context } \\
\text { Matching words from reading } \\
\text { to their definitions } \\
\text { Identifying reference words in } \\
\text { a text } \\
\text { Completing sentences with } \\
\text { missing words/phrases in } \\
\text { context } \\
\text { Matching sentence beginnings } \\
\text { to the endings } \\
\text { Matching statements to the } \\
\text { pictures } \\
\text { Discussing reading text } \\
\text { questions alone/in pairs }\end{array}$ \\
\hline
\end{tabular}




\begin{tabular}{|c|c|c|c|c|c|}
\hline & $\begin{array}{l}\text { order } \\
\text { Reading a text and writing a } \\
\text { letter } \\
\text { Predicting the end of story } \\
\text { Discussing reading text } \\
\text { questions alone/in pairs }\end{array}$ & & & & \\
\hline $\begin{array}{l}\text { Listening } \\
\text { tasks }\end{array}$ & $\begin{array}{l}\text { Understanding facts and details } \\
\text { Listening to words and } \\
\text { repeating } \\
\text { Listening for useful phrases } \\
\text { and repeating them loud and } \\
\text { completing the gap } \\
\text { Guessing missing words in } \\
\text { sentences, listening and } \\
\text { checking them } \\
\text { Answering rhetorical purpose } \\
\text { questions } \\
\text { Listening to dialogue and } \\
\text { filling a gap with missed words } \\
\text { Listening to dialogue and } \\
\text { practice it in speaking } \\
\text { Listening for } \\
\text { expressions/phrases and } \\
\text { finding meaning in native } \\
\text { language } \\
\text { Listening and completing a } \\
\text { table } \\
\text { Listening and following/complet } \\
\text { directions on the map } \\
\text { Predicting the end of story or } \\
\text { speaker's future message } \\
\text { Discussing } \\
\text { listening text questions } \\
\text { alone/in pairs }\end{array}$ & $\begin{array}{l}\text { Understanding main idea } \\
\text { Understanding facts and details } \\
\text { Inferring information from a } \\
\text { context } \\
\text { Listening and completing } \\
\text { sentences with missing } \\
\text { words/phrases } \\
\text { Matching statements/extracts to } \\
\text { pictures } \\
\text { Recognizing functions of } \\
\text { words/phrases/statements } \\
\text { Discussing } \\
\text { listening text questions } \\
\text { alone/in pairs }\end{array}$ & $\begin{array}{l}\text { Understanding main idea } \\
\text { Understanding facts and } \\
\text { details } \\
\text { Listening and completing } \\
\text { sentences/extracts with } \\
\text { missing words/phrases } \\
\text { Matching situations/extracts to } \\
\text { pictures } \\
\text { Listening and repeating } \\
\text { words/phrases } \\
\text { Discussing } \\
\text { listening text questions } \\
\text { alone/in pairs }\end{array}$ & $\begin{array}{l}\text { Understanding main idea } \\
\text { Understanding facts and } \\
\text { details } \\
\text { Understanding speaker's } \\
\text { attitude and purpose } \\
\text { Inferring information from a } \\
\text { context } \\
\text { Listening and completing } \\
\text { sentences with missing } \\
\text { words/phrases } \\
\text { Listening and completing } \\
\text { notes } \\
\text { Listen and matching } \\
\text { statements/extracts to pictures } \\
\text { Listening completing a table } \\
\text { Discussing } \\
\text { listening text questions } \\
\text { alone/in pairs }\end{array}$ & $\begin{array}{l}\text { Understanding main idea } \\
\text { Understanding facts and } \\
\text { details } \\
\text { Inferring information from a } \\
\text { context } \\
\text { Understanding speaker's } \\
\text { attitude and purpose } \\
\text { Repeating sentences } \\
\text { Perceiving and discriminating } \\
\text { individual sounds } \\
\text { Listening and ordering } \\
\text { sentences according to } \\
\text { recording } \\
\text { Recognizing functions of } \\
\text { phrases in context } \\
\text { Listening and competing } \\
\text { sentences/extracts with } \\
\text { missing words/phrases } \\
\text { Taking notes by filling a gap } \\
\text { in extracts } \\
\text { Discussing } \\
\text { listening text questions } \\
\text { alone/in pairs }\end{array}$ \\
\hline & Elementary & Elementary & Elementary & Elementary & Elementary \\
\hline $\begin{array}{l}\text { Reading } \\
\text { tasks }\end{array}$ & $\begin{array}{l}\text { Understanding facts and details } \\
\text { Inferring meaning of unknown } \\
\text { word in a context }\end{array}$ & $\begin{array}{l}\text { Understanding main idea } \\
\text { Understanding facts and details } \\
\text { Matching words/phrases to }\end{array}$ & $\begin{array}{l}\text { Understanding facts and } \\
\text { details } \\
\text { Completing sentences with }\end{array}$ & $\begin{array}{l}\text { Understanding facts and } \\
\text { details } \\
\text { Completing sentences }\end{array}$ & $\begin{array}{l}\text { Understanding main idea } \\
\text { Understanding facts and } \\
\text { details }\end{array}$ \\
\hline
\end{tabular}




\begin{tabular}{|c|c|c|c|c|c|}
\hline & $\begin{array}{l}\text { Completing sentences with } \\
\text { words/phrases } \\
\text { Completing a dialogue } \\
\text { Translating sentences into } \\
\text { native language } \\
\text { Completing a table with } \\
\text { grammar forms in the task } \\
\text { Matching headings to } \\
\text { paragraphs } \\
\text { Matching paragraphs to } \\
\text { pictures } \\
\text { Numbering paragraphs in } \\
\text { chronological order } \\
\text { Discussing reading text } \\
\text { questions alone/in pairs }\end{array}$ & $\begin{array}{l}\text { pictures } \\
\text { Completing sentences with } \\
\text { missing words/phrases in a } \\
\text { context } \\
\text { Recognizing/using correct } \\
\text { grammar points or grammar in } \\
\text { context } \\
\text { Discussing reading text } \\
\text { questions alone/in pairs }\end{array}$ & $\begin{array}{l}\text { missing words/phrases in } \\
\text { context } \\
\text { Matching statements to } \\
\text { pictures } \\
\text { Matching headings to } \\
\text { paragraphs } \\
\text { Matching sentence beginnings } \\
\text { to the endings } \\
\text { Summarizing information } \\
\text { Discussing reading text } \\
\text { questions alone/in pairs }\end{array}$ & $\begin{array}{l}\text { according to pictures } \\
\text { Matching statements to } \\
\text { pictures } \\
\text { Inferring meaning of } \\
\text { unknown word from a context } \\
\text { Translating the selected words } \\
\text { Completing sentences with } \\
\text { missing words/phrases in } \\
\text { context } \\
\text { Matching headings to } \\
\text { passages } \\
\text { Discussing reading text } \\
\text { questions alone/in pairs }\end{array}$ & $\begin{array}{l}\text { Completing sentences with } \\
\text { missing words/phrases in } \\
\text { context } \\
\text { Matching words from a text to } \\
\text { their definitions } \\
\text { Matching words to pictures } \\
\text { Matching titles to statements } \\
\text { Matching questions to } \\
\text { statements } \\
\text { Matching heading to } \\
\text { paragraphs } \\
\text { Identifying reference words in } \\
\text { a text } \\
\text { Recognizing/using grammar } \\
\text { points or grammar in context } \\
\text { Discussing reading text } \\
\text { questions alone/in pairs }\end{array}$ \\
\hline $\begin{array}{l}\text { Listening } \\
\text { tasks }\end{array}$ & $\begin{array}{l}\text { Understanding facts and details } \\
\text { Listen, read and match } \\
\text { situations/dialogues to pictures } \\
\text { Listening and completing a } \\
\text { dialogue } \\
\text { Listening and repeating a } \\
\text { dialogue } \\
\text { Perceiving and discriminating } \\
\text { sounds } \\
\text { Listening and completing a } \\
\text { table } \\
\text { Discussing } \\
\text { listening text questions } \\
\text { alone/in pairs }\end{array}$ & $\begin{array}{l}\text { Understanding facts and details } \\
\text { Listening and practicing } \\
\text { conversation } \\
\text { Listening and repeating words } \\
\text { Listening and completing } \\
\text { sentences/extracts with missing } \\
\text { words/phrases } \\
\text { Matching } \\
\text { situations/extracts/statements to } \\
\text { pictures } \\
\text { Listening and ordering } \\
\text { statements according to the } \\
\text { recording } \\
\text { Discussing } \\
\text { listening text questions } \\
\text { alone/in pairs }\end{array}$ & $\begin{array}{l}\text { Understanding facts and } \\
\text { details } \\
\text { Listening and repeating words } \\
\text { Listening and completing } \\
\text { sentences/extract with missing } \\
\text { words/phrases } \\
\text { Listening and ordering pictures } \\
\text { according to the recording } \\
\text { Matching statements/extracts } \\
\text { to pictures } \\
\text { Discussing } \\
\text { listening text questions } \\
\text { alone/in pairs }\end{array}$ & $\begin{array}{l}\text { Understanding facts and } \\
\text { details } \\
\text { Listen and repeating } \\
\text { words/sentences } \\
\text { Perceiving and discriminating } \\
\text { individual sounds } \\
\text { Listening to conversation and } \\
\text { repeating it in pairs } \\
\text { Listening and ordering } \\
\text { sentences according to the } \\
\text { recording } \\
\text { Listening and completing } \\
\text { sentences with missing } \\
\text { words/phrases } \\
\text { Listening and completing a } \\
\text { table } \\
\text { Listening an completing } \\
\text { instructions/directions on the } \\
\text { map } \\
\text { Discussing } \\
\text { listening text questions } \\
\text { alone/in pairs }\end{array}$ & $\begin{array}{l}\text { Understanding facts and } \\
\text { details } \\
\text { Summarizing information } \\
\text { Listening and completing } \\
\text { sentences with missing } \\
\text { words/phrases } \\
\text { Perceiving and discriminating } \\
\text { individual sounds } \\
\text { Listening and repeating words } \\
\text { Listening to partner and } \\
\text { finding places on the map } \\
\text { Listening and ordering } \\
\text { sentences according to the } \\
\text { recording } \\
\text { Taking notes and completing } \\
\text { a table } \\
\text { Listening to conversation and } \\
\text { composing sentences with } \\
\text { words from conversation } \\
\text { Discussing } \\
\text { listening text questions } \\
\text { alone/in pairs }\end{array}$ \\
\hline
\end{tabular}




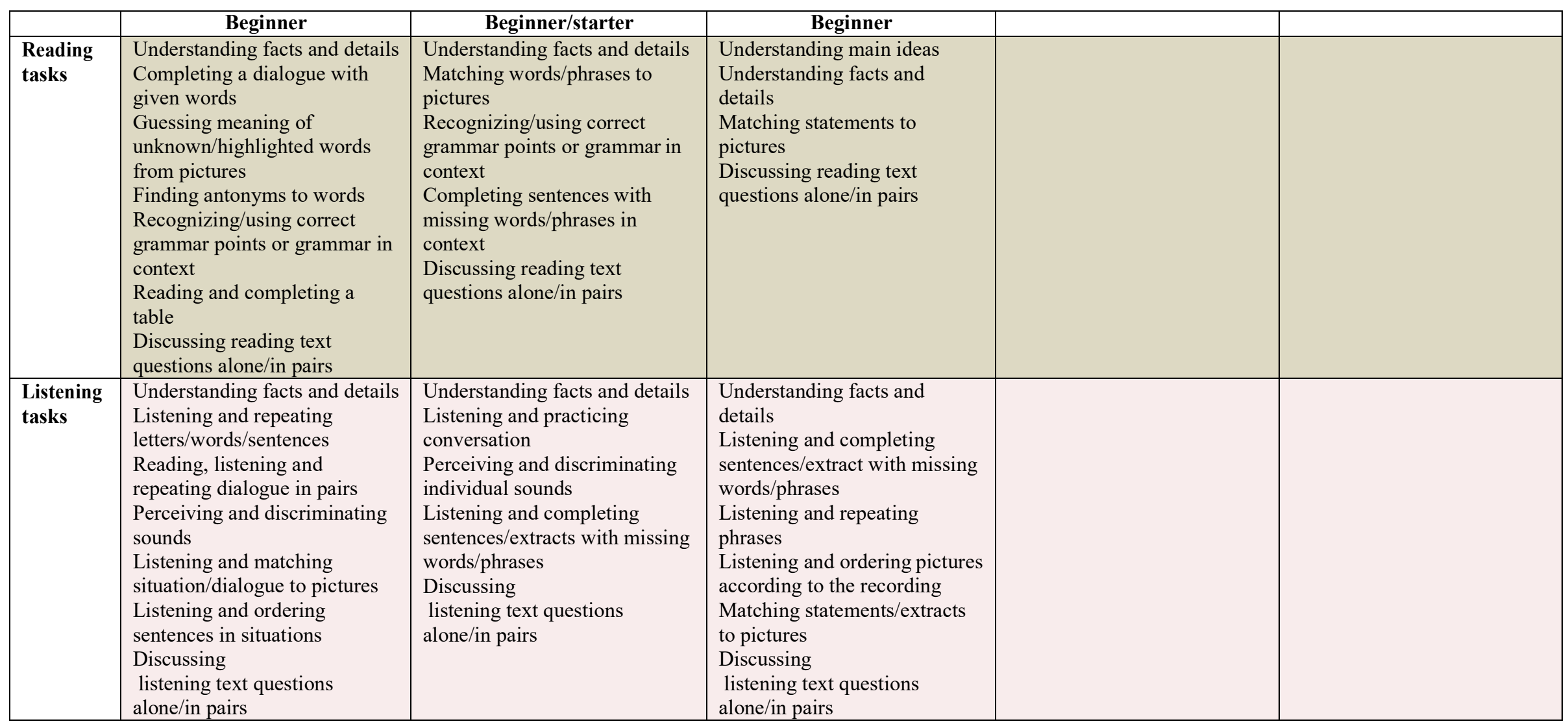

